\title{
Measurements of excitatory postsynaptic potentials in the stretch reflex of normal subjects and spastic patients
}

\author{
T. NOG UCH I, S. HOM M A, A N D Y. N A K A J I M A \\ From the Department of Physiology, School of Medicine, Chiba University, Chiba, Japan
}

SUMMARY The patellar tendon was tapped by random impulses of triangular waveform and motor unit spikes were recorded from the quadriceps femoris muscle. The cross-correlogram of the taps and the motor unit spikes revealed a primary correlation kernel, the width of which was interpreted as an indicator of the mean time-to-peak of excitatory postsynaptic potentials (EPSPs) elicited monosynaptically in an alpha-motoneurone by the triangular taps. The mean time-to-peak was $7.6 \pm 1.3 \mathrm{~ms}$ in normal subjects and $9.0 \pm 1.8 \mathrm{~ms}$ in spastic patients $(\mathrm{P}<0.005)$. The prolonged time-to-peak of EPSP in spastic patients is consistent with the hypothesis that as a result of degeneration of the corticomotoneuronal tract the Ia axons sprout and form more synaptic contacts on distal portions of the dendrites of alpha-motoneurones.

Brief stretching of a muscle by taps of triangular waveform and low amplitude can selectively excite primary endings of the muscle spindle. The primary spindle afferent impulses then ascend through group Ia nerve fibres to elicit ripples of excitatory postsynaptic potentials (EPSPs) in alpha-motoneurones (Homma et al., 1970; Homma, 1976). Continuous triangular form stretches result in temporal summation of the ripples of the EPSP. When the summated membrane potentials attain a critical firing level, the alpha-motoneurones fire. The spike potential of a motoneurone elicited by triangular stretch always occurs during the rising phase of the EPSP. Thus motor unit spikes appear within the time-to-peak of EPSPs. Since motoneurone spikes occur most frequently during the steepest rising slope of the EPSP and least frequently on the lower slopes either at the start or near the summit of the EPSP, the time course of an EPSP can be calculated from a probability density distribution of motor unit spikes obtained from a cross-correlation function between the motor unit spikes and the stimulus (Homma and Nakajima, 1979a, b).

The cross-correlogram of motor unit spikes with respect to the triangular stretch reveals one

Address for reprint requests: Dr S. Homma, Department of Physiology, School of Medicine, Chiba University, 1-8-1, Inohana, Chiba 280, Japan.

Accepted 23 May 1979 peak, termed the primary correlation kernel (Knox, 1974). The width of the kernel, the correlation time, corresponds to the time-to-peak of an EPSP elicited by the triangular stretch.

Since the relationship between the time-to-peak of an EPSP and excitatory synaptic terminals on dendrites or soma of a neurone is well known (Rall et al., 1967), we attempted to calculate the time-to-peak of an EPSP in normal subjects and in spastic patients, and to discuss the pathogenesis of the spasticity.

\section{Methods}

Responses to vibration were elicited in the triceps surae and quadriceps femoris muscles of 20 healthy men and 18 patients who showed spasticity. When responses in the triceps surae muscle were examined, the subject lay prone on a bed, and the malleoli were fixed in a rigid metal frame to reduce leg movements. The Achilles tendon was tapped by a vibrator located perpendicularly to it. The isometric tension of the triceps surac muscle was measured by a load cell attached to the plantar side of the foot. To examine responses of the quadriceps femoris the subject sat in a chair, while the hip and knee joint angles were set at $120^{\circ}$ and $90^{\circ}$, respectively. The knee joint was fixed to eliminate any movements of the leg. The patellar tendon was tapped by a vibrator located 
anterior to it. The isometric tension of the quadriceps femoris muscle was recorded by a load cell attached to the anterior side of the lower leg. Tendons were tapped by triangular shaped pulses with a rising and falling time of $4 \mathrm{~ms}$. Intervals between the pulses were changed randomly. The minimum and maximum interval were $20 \mathrm{~ms}$ and $80 \mathrm{~ms}$, respectively. The amplitude of the taps was adjusted from 400 to $600 \mu \mathrm{m}$.

Motor unit spikes were recorded by copper wire electrodes of $70 \mu \mathrm{m}$ diameter inserted into the muscle being examined. The isometric tension, the triangular taps, and the motor unit spikes were stored on magnetic tape for subsequent computation. The cross-correlogram between the motor unit spikes and triangular taps was calculated by computer (ATAC 501-20, Nihon Kohden, and HITAC 10-II, Hitachi Co).

\section{Results}

CROSS-CORRELOGRAM OF MOTOR UNIT SPIKES AND RANDOM TRIANGULAR TAPS

Random triangular taps to the Achilles tendon reflexly elicited motor unit spikes in the gastrocnemius muscle as shown in Fig. 1.

"A" indicates the motor unit spikes of the gastrocnemius muscle elicited by the random triangular taps, "Stim". Homma (1976) reported from intracellular recordings of the cat motoneurone that the primary spindle afferent impulses elicited by the taps produce EPSP ripples on the alpha-motoneurone and that the EPSP ripples show temporal summation. When the summated EPSP is sufficiently intense, the alphamotoneurone generates impulses which descend via the motor axon to excite the inncrvated muscle. Such reflex activity can be recorded as a motor unit spike.

Figure 1B is a cross-correlogram of motor unit spikes and triangular taps. The prominent peak in the cross-correlogram is called the primary correlation kernel. Since the primary correlation kernel corresponds with the probability distribution of the motor unit spikes which arise during the rising phase of the EPSPs, the distribution width of the kernel indicatcs the time-to-peak of the EPSP. The mean width of the primary cor-

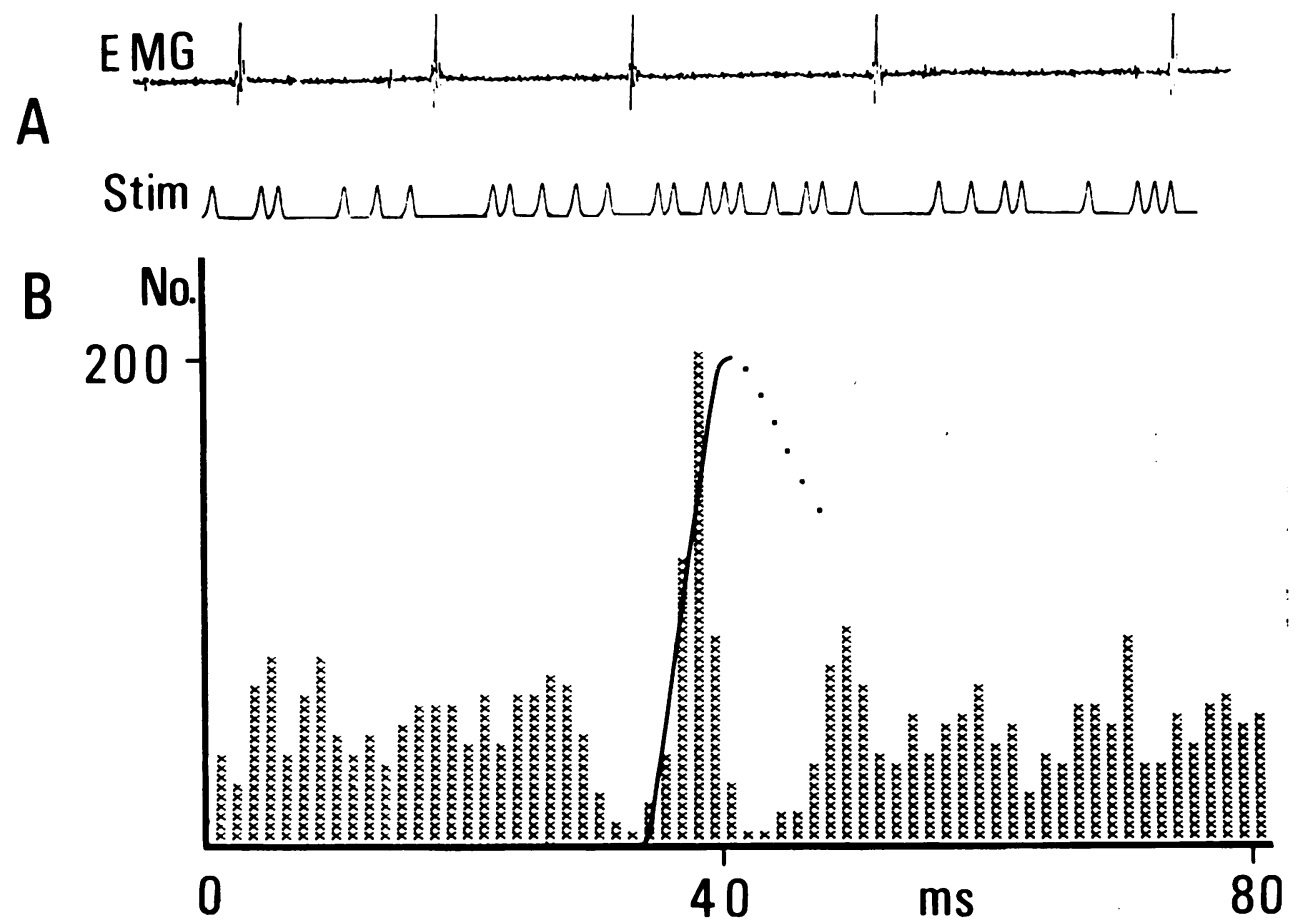

Fig. 1 A: motor unit spikes and random triangular waveform taps of the gastrocnemius muscle. B: cross-correlogram of the motor unit spikes and triangular taps. The prominent peak in the correlogram is called the primary correlation kernel. The kernel was integrated and is shown by a solid line. Because of the random intervals of the triangular taps, secondary correlation kernels form a plateau and are not evident. 
relation kernel, the correlation time, in 57 units of the gastrocnemius was $8.2 \pm 1.7 \mathrm{~ms}$. The primary correlation kernel was integrated and fitted to the following equation (Knox, 1974) by means of the least mean square.

$$
\begin{gathered}
\mathrm{Y}=\mathrm{t} p \cdot \exp \left(\begin{array}{c}
-p \cdot \mathrm{t} \\
\mathrm{CT}
\end{array}\right) \\
\mathrm{CT}: \text { correlation time } \\
p: \text { power }
\end{gathered}
$$

The integrated kernel is shown by a solid line in Fig. 1B. The solid line rises slowly after the onset, then becomes very steep and slows down again near the summit. Therefore, the line appears to represent the time course of the rising phase of an EPSP. The falling phase of the EPSP is shown by dots, because they are only based on calculation by the above formula. Secondary correlation kernels obtained by cross-correlation are not easily detected because as the triangular taps are random (Fig. 1B) they form a plateau. The latency to the onset of the primary correlation kernel reflects the time from the onset of the tap to the resultant motor unit spike. It is the so-called response time. The mean value for the minimum response time of the gastrocnemius muscle was $33.9 \pm 1.3 \mathrm{~ms}$ in 57 units. Since the response time of an $\mathrm{H}$ wave elicited by electrical stimulation of the tibial nerve at the popliteal fossa is about $30 \mathrm{~ms}$ (Magladery, 1955), it is likely that the motor unit spikes evoked by tap also represent a monosynaptic response of the stretch reflex.

The correlation time of man-that is, $8.2 \mathrm{~ms}-$ is very long when compared with the results obtained from the experiments on cats, in which the mean response and correlation time were $3.2 \pm 0.4 \mathrm{~ms}$ and $4.7 \pm 1.1 \mathrm{~ms}$, respectively (Homma and Nakajima, 1979b). We measured the mean response time and correlation time in 54 units of the quadriceps femoris muscles. The results are shown in Table 1, together with those obtained from the triceps surae muscles.

Table 1 Mean response and correlation time in gastrocnemius and quadriceps femoris muscles

\begin{tabular}{lll}
\hline $\begin{array}{l}\text { Muscle } \\
\text { Gastrocnemius } \\
\quad(57 \text { units) }\end{array}$ & Response time $(m s)$ & Correlation time $(\mathrm{ms})$ \\
$\begin{array}{l}\text { Quadriceps femoris } \\
\quad(54 \text { units) }\end{array}$ & $20.4 \div 3.4 \mathrm{SD}$ & $8.2 \pm 1.7 \mathrm{SD}$ \\
\end{tabular}

The shorter response time in the quadriceps femoris muscle than in the gastrocnemius muscle is due to the fact that the reflex arc to the former is shorter than to the latter. The correlation time is almost equal in both muscles.

\section{CROSS-CORRELOGRAMS IN NORMAL SUBJECTS ANI)} PATIENTS WITH SPASTICITY

The patellar tendon was tapped by the random triangular pulses and the motor unit spikes were recorded from the quadriceps femoris muscle in normal men and patients with spasticity.

The left half of Fig. 2 shows records obtained from a normal subject and the right half from a spastic patient. On the upper trace, motor unit
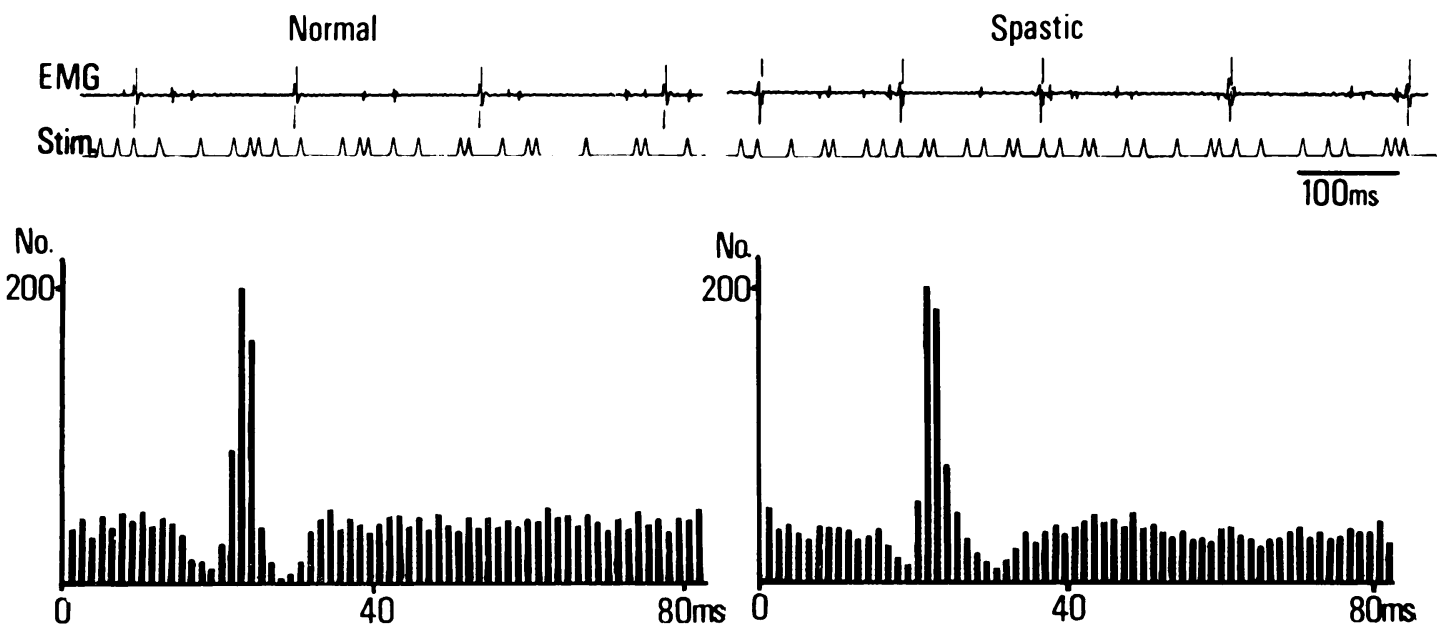

Fig. 2 Cross-correlograms of the quadriceps femoris motor unit spikes in a normal subject (left) and a spastic patient (right). From the upper trace downward, motor unit sp:kes; random triangular taps; cross-correlogram of the motor unit spikes and the random triangular taps. 


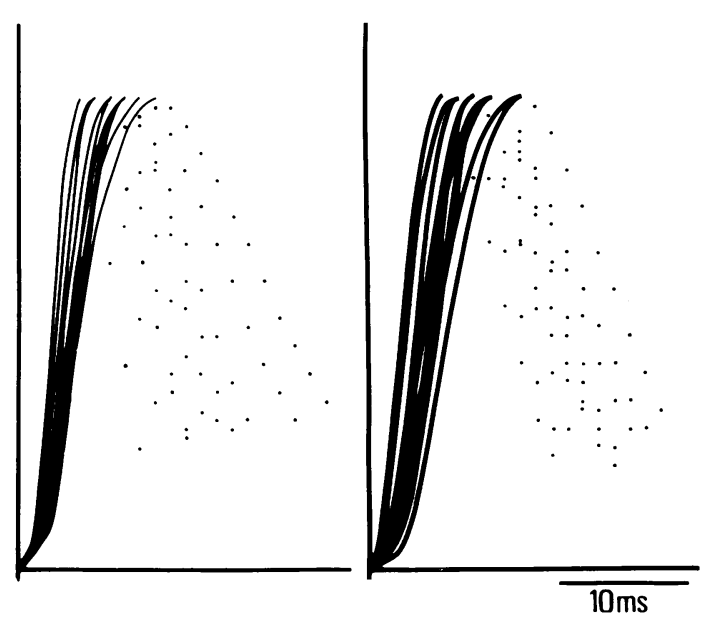

Fig. 3 Integrated curves of primary correlation kernels recorded from the quadriceps femoris in 10 normal subjects (left) and 10 spastic patients (right). See text for details.

spikes, random triangular taps, and the crosscorrelograms between the motor unit spikes and the random triangular taps are shown. Both correlograms show the primary correlation kernel. Ten examples of the kernel in each case were integrated and superimposed (Fig. 3).

The left half of Fig. 3 represents results obtained from normal subjects, and the right half results obtained from spastic patients. All of the curves begin with a slowly rising phase followed by a steep phase and end with a slowly rising curve. Thus, these curves reflect the rising phase of EPSPs.

The rising phase of EPSPs in spastic patients is slower than in normal subjects. The mean values of the time-to-peak were measured in 26 normal men and 18 patients with spasticity (Table 2 ).

The mean value of time-to-peak in 32 units of normal subjects is $7.6 \pm 1.3 \mathrm{~ms}$ and in 31 units of spastic patients $9.0 \pm 1.8 \mathrm{~ms}$. The time-to-peak of the spastic patients is longer than that of normal subjects with statistical significance $(P<0.005)$.

Table 2 Mean values of time-to-peak of EPSP in quadriceps femoris muscle

\begin{tabular}{ll}
\hline & Correlation time $(m s)$ \\
\hline $\begin{array}{l}\text { Normal subjects } \\
(32 \text { units) }\end{array}$ & $7.6 \pm 1.3$ \\
$\begin{array}{c}\text { Spastic patients } \\
\text { (31 units) }\end{array}$ & $9.0 \pm 1.8^{*}$ \\
\hline
\end{tabular}

*statistically significant $(\mathrm{P}<0.005)$

\section{Discussion}

When a muscle is stretched by triangular waveform taps, ripples of EPSPs corresponding to the triangular stretches are seen in an alpha-motoneurone (Homma et al., 1970; Homma, 1976). Continuous application of the triangular stretches causes temporal summation of the ripples of EPSPs, and when the summated membrane potential attains the critical firing level, the alphamotoneurone discharges (Homma, 1976). At all times, the motoneurone spikes occur during the rising phase of the EPSPs-that is, within the time-to-peak of the EPSPs. Furthermore, these spikes occur most frequently at the steepest rising slope of the EPSPs and least frequently on the slower slopes both at the start and near the summit of the EPSPs. The critical threshold level for firing of the motoneurone accommodates to the rising slope of the EPSPs. Thus the less steep the slope of the EPSP, the higher the firing level (Homma, 1966; Sasaki and Otani, 1961). Because of this, the motoneurone has a tendency to fire at the steepest portion of the rising slope of the EPSPs. These facts led us to conclude that the time course of the EPSP can be calculated from a probability distribution of motor unit spikes which appear in the cross-correlogram of motor unit spikes and stretch pulses (Homma and Nakajima, 1979b). The cross-correlation showed a prominent kernel, the primary correlation kernel. Using a neurone model, Knox in 1974 demonstrated that the width of the kernel, the correlation time, is related to the derivatives of postsynaptic potentials. Our experimental results strongly support his theoretical point of view.

When the stretch pulses are delivered with a constant frequency, periodic secondary kernels appear in the cross-correlogram coinciding with the cycle time of the stimuli (Homma and Nakajima, 1979a). These secondary kernels make it difficult to distinguish the primary correlation kernel. In contrast, when the stretch pulses are delivered randomly, the secondary correlation kernels become a plateau making the primary correlation kernel obvious (Knox, 1974; Homma and Nakajima, 1979b). For these reasons, in our study the stimulus intervals were changed randomly. The minimum interstimulus interval was set at $20 \mathrm{~ms}$, a time selected because it needs to be longer than the correlation time. The maximum interval was $80 \mathrm{~ms}$, which was adjusted to the total sampling time of the cross-correlation analysis. Thus, stretch intervals were changed randomly from 20 to $80 \mathrm{~ms}$ with uniform probability distribution. 
In these experiments, we studied whether or not this statistical measurement is applicable in calculating the time-to-peak of an EPSP elicited in the human stretch reflex. Vibratory stimuli to a muscle cause tonic contraction in man. This phenomenon is the "tonic vibration reflex" (TVR) (Hagbarth and Eklund, 1966). Motor unit spikes obtained from the contracting muscle are phaselocked to the vibratory stimuli in the initial stage of the contraction. Thus, the frequency of the phase-locked spikes is decoded $1 / n$ with respect to the vibratory frequency $(\mathrm{n}=$ multiple integer $)$ (Homma et al., 1972; Homma and Kanda, 1973). This implies that motoneurones fire as a result of the temporal summation of EPSP ripples elicited by the vibratory stimuli and that the motoeneurone spikes always take place during the rising phase of the EPSP.

It is necessary to consider how selectively primary spindle endings are activated by muscle vibration in man. Bianconi and Van der Meulen (1963) and Matthews (1967) found in cats that the primary endings can follow sinusoidal vibration of the muscle up to higher frequencies than can the secondary endings. The relative sensitivity of muscle receptors to sinusoidal muscle stretch was higher in the primary endings, less in the Golgi tendon organs, and least in secondary endings (Fukuda, 1972). Thus, only the primary endings can respond to a fast rise time of muscle stretch (Tamaki, 1967). These observations suggest that it is the primary endings which are most sensitive to quick stretch of a muscle.

The maximal contraction tension during the TVR was obtained in man by sinusoidal muscle vibration of $100-120 \mathrm{~Hz}$. Even in the case of the tendon reflex in man, abrupt tap of a tendon elicits intense reflex contraction. In cats the quick stretch of a muscle elicits a steeper EPSP in an alpha-motoneurone than does slow stretch, and the steeper the EPSP, the lower the critical firing level of the alpha-motoneurone (Homma, 1966). On this basis we have proposed (Homma, 1976) that tendon taps with a triangular waveform which have a rising phase of $4 \mathrm{~ms}$ excite primary endings and can elicit a powerful stretch reflex.

The first reason why the stimulus intervals were changed randomly in this study was to eliminate the formation of the secondary kernels in the cross-correlogram. The second was that, since the derivative of the EPSP is statistically computed as a probability distribution of the motor unit spikes, it is desirable that the probability with which the EPSP crosses the firing level is determined uniformly. Random taps which elicited more than 200 motor unit spikes showed uniform distribution in the interval histogram. The widths of the primary correlation kernels obtained from the crosscorrelograms of the random taps and the motor unit spikes of more than two hundred units were of the same value. Nevertheless, in this study more than 400 motor unit spikes were analysed in one trial and the widths of the primary correlation kernels were measured.

The mean value of the time-to-peak of the EPSPs in normal subjects was $7.6 \mathrm{~ms}$ and in spastic patients $9.0 \mathrm{~ms}$. The time-to-peak of the spastic patients was longer than in normal subjects with statistical significance. Rall et al. (1967) made the theoretical suggestion that the time-to-peak of an EPSP becomes longer if the input terminals make synaptic contacts with the more distal part of the dendrites of a neurone. Since the descending pathways to alpha-motoneurones form their synapses at more distal parts of the dendrites than do the primary spindle afferents fibres, the time-topeak of corticomotoneuronal EPSPs is longer than that for Ia afferent fibres (Porter and Hore, 1969; Shapovalov, 1975).

Degeneration of the descending corticospinal pathways causes collateral sprouting from afferent nerves to the motoneurone, and the new sprouts form synapses upon synaptic sites previously oc- $\stackrel{\mathbb{Q}}{\triangle}$ cupied by degenerating descending pathways (Mc- -0 Couch et al., 1958). In the central nervous system, $\infty$ such degeneration of an input causes collateralo sprouting of the surviving input axons (Tsukahara et al., 1974; Goldberger and Murray, 1978; Precht and Dierenger, 1979). The sprouting is regarded as a compensatory function of the nervous system.

On the basis of these observations it may be concluded that prolongation of the time-to-peak of the EPSP in spastic patients is probably the result of temporal summation of a new EPSP with a long time-to-peak, elicited by the sprouting of the primary spindle afferent fibres to the degenerated sites of distal dendrites of alpha-motoneurones.

\section{References}

Bianconi, R., and Van der Meulen, J. P. (1963). The response of the end-organs of mammalian muscle spindles. Journal of Physiology, 26, 177-190.

Fukuda, K. (1972). Ia, Ib and II discharges during muscle vibration. Journal of the Physiological Society of Japan, 35, 242-250.

Goldberger, M. E., and Murray, M. (1978). Recovery of movement and axonal sprouting may obey some of the same laws. In Neural Plasticity, pp. 73-95. Edited by C. W. Cotman. Raven Press: New York.

Hagbarth, K. E., and Eklund, G. (1966). Motor effects of vibratory muscle stimuli in man. In 
Muscular Afferents and Motor Control, pp. 177186. Edited by R. Granit. Almqvist and Wiksell: Stockholm.

Homma, S. (1956). Firing of the cat motoneurone and summation of the excitatory postsynaptic potential. In Muscular Afferents and Motor Control, pp. 235244. Edited by R. Granit. Almqvist and Wiksell: Stockholm.

Homma, S. (1976). Frequency characteristics of the impulse decoding ratio between the spinal afferents and efferents in the stretch reflex. In Understanding the Stretch Reflex. Progress in Brain Research, vol. 44, pp. 15-30. Edited by S. Homma. Elsevier: Amsterdam.

Homma, S., and Nakajima, Y. (1979a). Coding process in human stretch reflex analyzed by phaselocked spikes. Neuroscience Letters, 11, 19-22.

Homma, S., and Nakajima, Y. (1979b). Input-output relation in spinal motoneurons during the stretch reflex. In Progress in Brain Research, vol. 50. Elsevier: Amsterdam. In press.

Homma, S., and Kanda, K. (1973). Impulse decoding process in stretch reflex. In Motor Control, pp. 45-64. Edited by A. A. Gydikov, N. T. Tankov, and D. S. Kosarov. Plenum Press: New York.

Homma, S., Ishikawa, K., and Stuart, D. G. (1970). Motoneuron responses to linearly rising muscle stretch. American Journal of Physical Medicine, 49, 290-306.

Homma, S., Kanda, K., and Watanabe, S. (1972). Preferred spike intervals in the vibration reflex. Japanese Journal of Physiology, 22, 421-432.

Knox, C. K. (1974). Cross-correlation functions for a neuronal model. Biophysical Journal, 14, 567-582.

McCouch, G. P., Austin, G. M., Liu, C. M., and Liu, C. Y. (1958). Sprouting as a cause of spasticity. Journal of Neurophysiology, 21, 205-216.
Magladery, J. W. (1955). Some observations on spinal reflexes in man. Pfü̈gers Archiv fur die Gesamte Physiologie des Menschen und der Tiere, 261, 302321.

Matthews, P. B. C. (1967). Vibration and the stretch reflex. In Myotatic, Kinesthetic and Vestibular Mechanisms, pp. 40-50. Edited by A. V. S. De Reuck and J. Knight. Churchill: London.

Porter, R., and Hore, J. (1969). Time course of minimal corticomotoneuronal excitatory postsynaptic potentials in lumbar motoneurons of the monkey. Journal of Neurophysiology, 32, 443-451.

Precht, W., and Dieringer, N. (1979). Synaptic mechanisms involved in compensation of vestibular function following hemilabyrinthectomy. In Progress in Brain Research, vol. 50. Elsevier: Amsterdam. In press.

Rall, W., Burke, R. E., Smith, T. G., Nelson, P. G., and Frank, K. (1967). Dendritic location of synapses and possible mechanisms for the monosynaptic EPSP in motoneurons. Journal of Neurophysiology, 30, 1169-1193.

Sasaki, K., and Otani, T. (1961). Accommodation in spinal motoneurones of the cat. Japanese Journal of Physiology, 11, 443-456.

Shapovalov, A. I. (1975). Neuronal organization and synaptic mechanisms of supraspinal motor control in vertebrates. Review of Physiology, Biochemistry, and Pharmacology, 72, 1-54.

Tamaki, T. (1967). Muscle spindle and tendon organ discharge during phasic muscle stretching. Journal of Chiba Medical Society, 43, 649-657.

Tsukahara, N., Hultborn, H., and Murakami, F. (1974). Sprouting of cortico-rubal synapses in red nucleus neurons after destruction of the nucleus interpositus of the cerebellum. Experient:a, 30, $57-58$. 